Supplement of Biogeosciences, 16, 4783-4803, 2019

https://doi.org/10.5194/bg-16-4783-2019-supplement

(C) Author(s) 2019. This work is distributed under

the Creative Commons Attribution 4.0 License.

(c) (1)

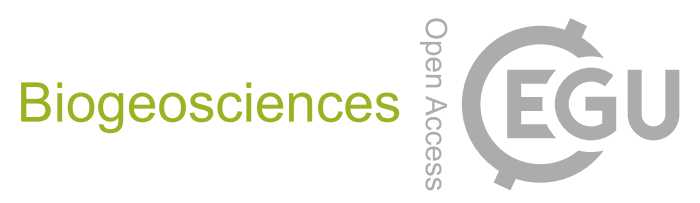

Supplement of

\title{
Reviews and syntheses: Agropedogenesis - humankind as the sixth soil- forming factor and attractors of agricultural soil degradation
}

Yakov Kuzyakov and Kazem Zamanian

Correspondence to: Yakov Kuzyakov (kuzyakov@gwdg.de) and Kazem Zamanian (zamanians@yahoo.com)

The copyright of individual parts of the supplement might differ from the CC BY 4.0 License. 


\section{History of the equation $S=f(c l, o, r, p, t, \ldots)$ in:}

Florinsky, I.V. 2012. The Dokuchaev hypothesis as a basis for predictive digital soil mapping (on the 125th anniversary of its publication). Eurasian Soil Science 45 (4), 445-451.

(see full paper at the end of the Supplement).

The "Jenny" state factor equation for soil genesis: $S=f(C, O, R, P, T, \ldots)$ was copy-pasted from the textbook for Russian students published in 1927 by S.A. Zakharov:

Zakharov, S. A., 1927. A Course of Soil Science. Gosizdat, Moscow - Leningrad. [in Russian: Захаров C.A., 1927. Курс Почвоведения. Государственное Издательство Москва - Ленинград].

where $\Pi$ is soil, $\mathrm{K}$ is climate, $\mathrm{O}$ is organisms, $\Gamma$ is parent material, and B is the age of the soil. Topography was not included into the expression probably due to a stenographer's mistake, because Eq. (3) is preceded by two sentences discussing the important role of topography in soil formation (Fig. 2).

In 1927, Zakharov presented a general soil formation equation in a well-known fundamental textbook $[35$, p. 8] (Fig. 3). This equation ideally described the Dokuchaev postulate:

$$
\pi=f(\text { М.Г.П.,Р.Ж.Орг.,Кл., Возр.стр.,Р-ф), }
$$

where $\pi$ is soil, М.Г.П. is parent rock material, Р.Ж.Орг. is plant and animal organisms, Кл. is climate, Возр. стр. is the age of the terrain, and $\mathrm{P}-\phi$ is topography. There were three misprints in Eq. (4): two commas were missed and there was an excess close bracket (Fig. 3). The misprints were fixed in the second edition [36, p. 18]. Unlike the rare brochure [8], textbooks $[35,36]$ were widespread $(4000$ and 18000 copies of the first and second editions were printed, respectively).

In the summer of 1927 , the Dokuchaev hypothesis where $S$ is soil, $M$ is parent material, $C$ is climate, $V$ is vegetation, $T$ is time, and $D$ is erosion or deposition. Shaw did not mention either the Dokuchaev hypothesis or the equations of Dokuchaev (3) and Zakharov (4). It is interesting that Shaw presented Eq. (5) at the Second International Congress of Soil Science held in the USSR in 1930 [27]. Zakharov took part in the discussion of this presentation. He criticized Shaw for ignoring the role of topography, animals, and humans in Eq. (5) [27, p. 14].

In 1941, Wilde published his equation [33, p. 34]:

$$
S=\int(\text { g.e.b. }) d t,
$$

where $S$ is soil, $g$ is geological substratum, $e$ is the environmental influences, $b$ is the biological activity, and $t$ is time. Wilde did not mention Eqs. (3), (4), and (5). Note that Wilde, a Russia-born immigrant, was familiar with the works of Dokuchaev and Zakharov. Indeed, he cited the Dokuchaev postulate right before Eq. (6) calling it "the first basic law of soil science" (Zakharov's expression [35, p. 8]).

In 1941, Jenny published Eq. (2) [16, p. 16] and selectively cited the Dokuchaev hypothesis (after Afanasiev [1. v. 101). that is. onlv a portion of the limiting 


\section{Fig. S1: Soil depth decrease due to erosion.}

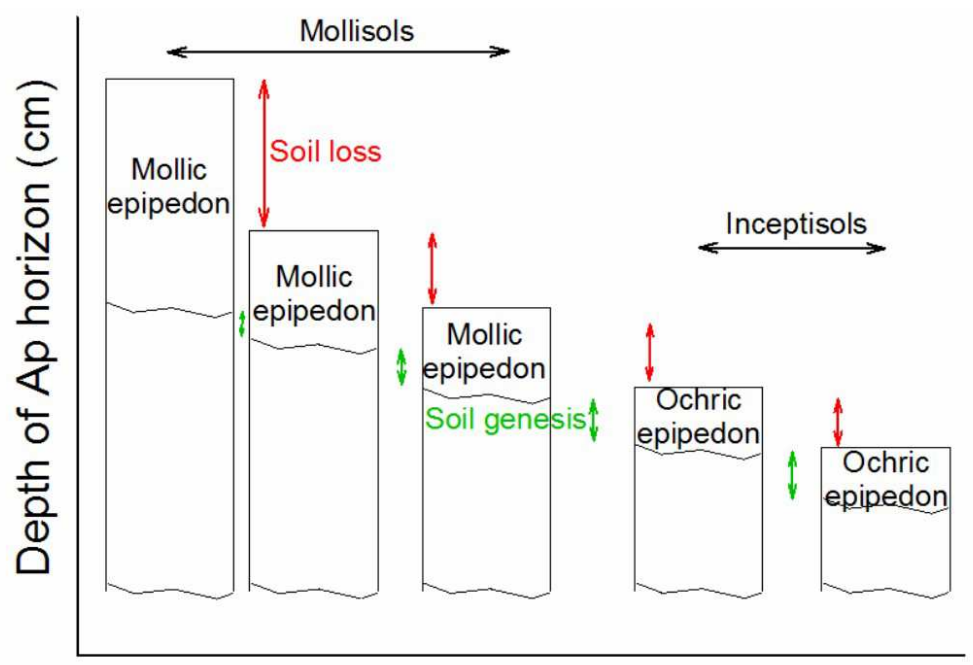

Cultivation duration (years)
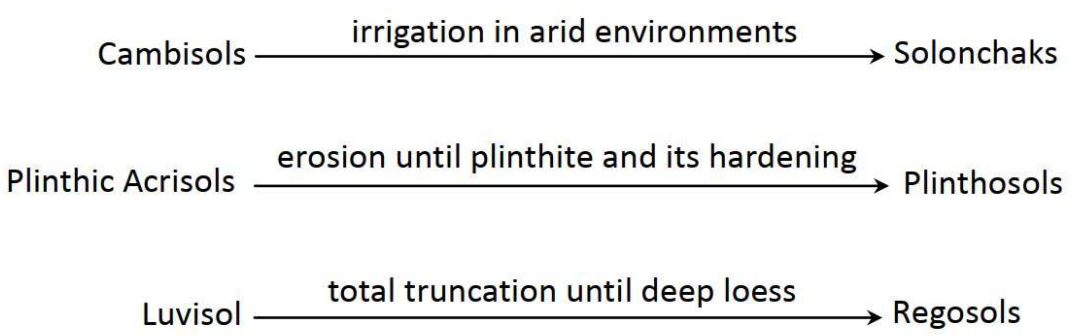

Haplic Nitisols $\stackrel{\text { surface waterlogging with long lasting }}{\text { rice irrigation }}$ Anthraquic Nitisols

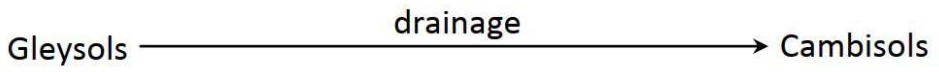

Protothionic Fluvisols $\longrightarrow$ drainage $\longrightarrow$ Orthithionic Fluvisols

Supplementary Fig. 1: Soil depth decrease due to erosion. The erosion rate decreases with cultivation duration due to depletion of easily erodible materials. It reaches steady state conditions when erosion becomes equal to soil genesis. After major erosion, the soil taxonomic group changed due to a strong decrease in the Ah / Ap horizon depth, which led to new qualifiers and master properties. Other frequent examples of soil class changes are presented in Dudal (2004). 


\section{Fig. S2: Convergence of soil properties as a result of cultivation duration.}
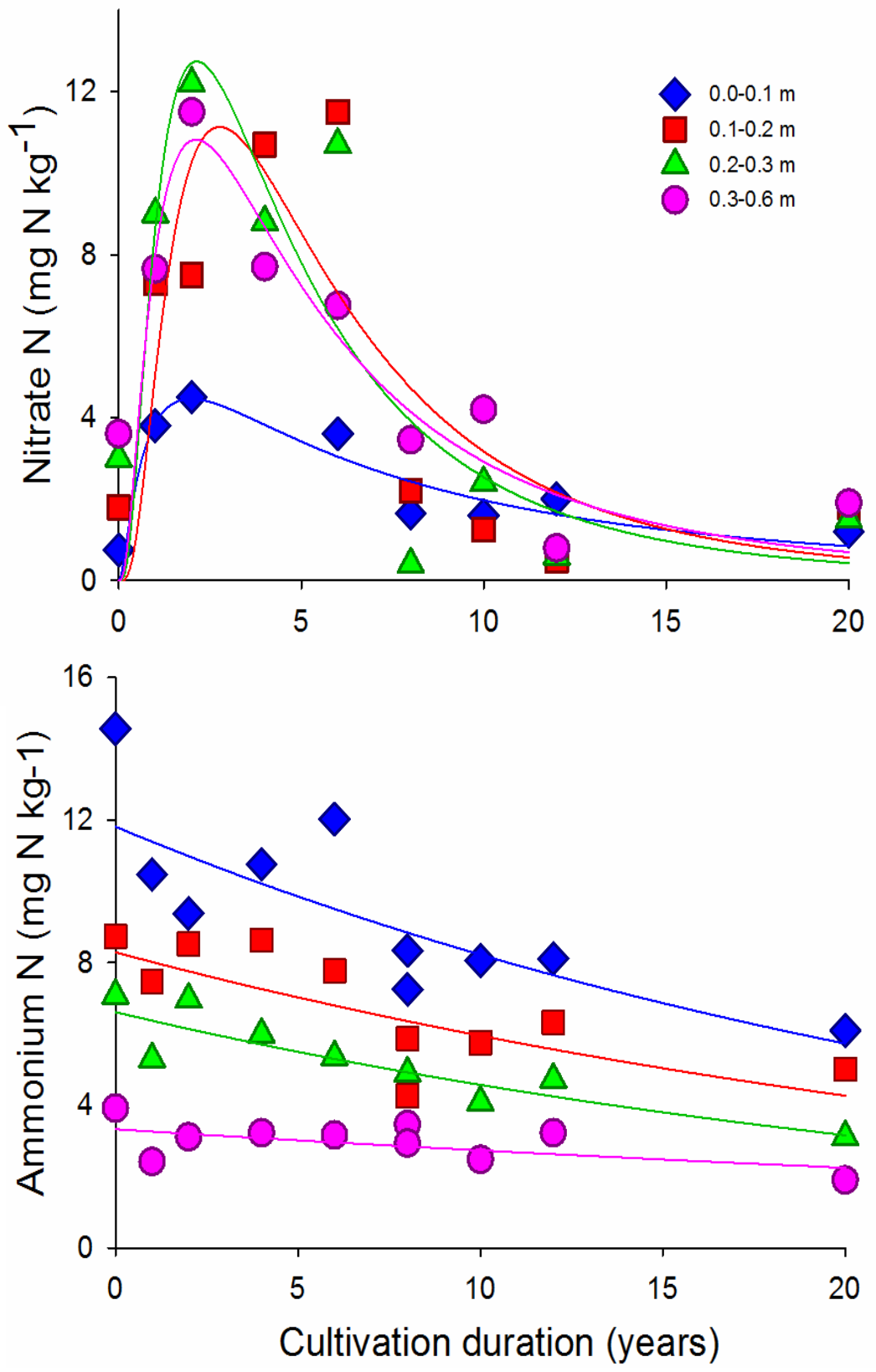

Supplementary Fig. 2: Examples of convergence of soil properties as a result of cultivation duration: (top) Nitrate content, (bottom) ammonium content depending on soil depth during 20 years of cultivation (Jones and Dalal, 2017). The solid lines are added to better visualize the changing trends in nitrate and ammonium contents as a function of cultivation duration. 


\section{Fig. S3: Convergence of soil properties as a result of cultivation duration.}

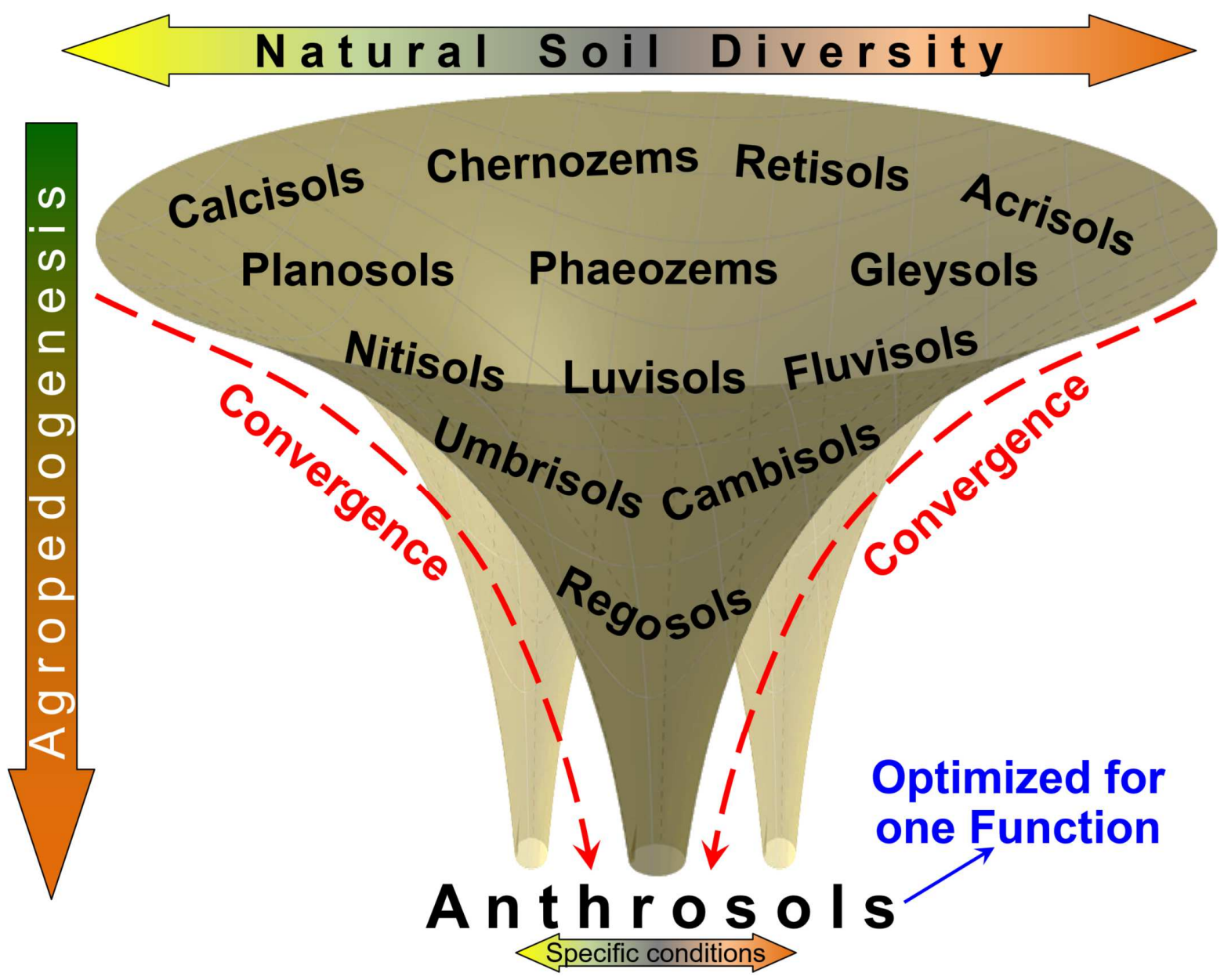

Supplementary Fig. 3: Extended conceptual schema of convergence of soil properties by agropedogenesis (see also Fig. 9). The very broad range of natural soils and their properties will be tailored for crop production by agricultural use, resulting in Anthrosols with a very narrow range of properties - the convergence of properties by agropedogenesis. Note that the soils within the funnel are mentioned exemplarily and not all WRB soil groups are presented. The sequence of soils within the funnel does not reflect their transformations during agropedogenesis to Anthrosols. This extended version reflecting multiple pathways to Anthrosols and their variability. Nevertheless, the variability of all soil parameters is much lower compared to natural soils. 


\title{
The history of the equation $S=f(c l, o, r, p, t, \ldots)$
}

ISSN 1064-2293, Eurasian Soil Science, 2012, Vol. 45, No. 4, pp. 445-451. (C) Pleiades Publishing, Ltd., 2012.

Original Russian Text ( I.V. Florinsky, 2012, published in Pochvovedenie, 2012, No. 4, pp. 500-506.

\section{The Dokuchaev Hypothesis as a Basis for Predictive Digital Soil Mapping (On the 125th Anniversary of Its Publication)}

\author{
I. V. Florinsky \\ Institute of Mathematical Problems of Biology, Russian Academy of Sciences, Pushchino, Moscow oblast, 142290 Russia \\ E-mail: iflor@mail.ru
}

Received June 16, 2011; in final form, September 25, 2011

\begin{abstract}
Predictive digital soil mapping is widely used in soil science. Its objective is the prediction of the spatial distribution of soil taxonomic units and quantitative soil properties via the analysis of spatially distributed quantitative characteristics of soil-forming factors. Western pedometrists stress the scientific priority and principal importance of Hans Jenny's book (1941) for the emergence and development of predictive soil mapping. In this paper, we demonstrate that Vasily Dokuchaev explicitly defined the central idea and statement of the problem of contemporary predictive soil mapping in the year 1886. Then, we reconstruct the history of the soil formation equation from 1899 to 1941 . We argue that Jenny adopted the soil formation equation from Sergey Zakharov, who published it in a well-known fundamental textbook in 1927. It is encouraging that this issue was clarified in 2011, the anniversary year for publications of Dokuchaev and Jenny.
\end{abstract}

DOI: $10.1134 / \mathrm{S} 1064229312040047$

\section{INTRODUCTION}

In soil science, predictive digital soil mapping has received wide recognition in the past twenty years [5, $12,13,22]$. Its object is the prediction of the spatial distribution of (1) soil taxonomic units and (2) the physical, chemical, and biological quantitative properties of soils. The prediction is based on the analysis of the spatially distributed quantitative characteristics of the soil forming factors using various mathematical techniques, such as multiple regression analysis, hybrid geostatistical approaches, fuzzy logic, discriminant analysis, artificial neural networks, etc.

As a theoretical basis for predictive soil mapping, McBratney et al. [24] proposed SCORPAN, a model for empirical quantitative descriptions of relationships between soils and spatially distributed predictors:

$$
S_{c}=f(s, c, o, r, p, a, n), S_{a}=f(s, c, o, r, p, a, n),
$$

where $S_{c}$ is soil classes, $S_{a}$ is soil attributes, $s$ is soil (other properties of the soil at a given point), $c$ is climate (local climatic properties), $o$ is organisms (vegetation, fauna, and human activity), $r$ is relief (topography, morphometric variables), $p$ is parent material (lithology), $a$ is age (time), and $n$ is space (spatial position). The authors of SCORPAN noted that Eqs. (1) are versions of the well-known equation of Hans Jenny, which describes soil as a function of soil forming factors [16, p. 16]:

$$
S=f(c l, o, r, p, t, \ldots),
$$

where $S$ is soil; and $c l, o, r, p$, and $t$ are soil-forming factors (climate, organisms, topography, parent material, and time, respectively). The ellipsis indicates that additional soil formers may be included in Eq. (2). McBratney et al. [24, p. 6] stressed the scientific priority and principal importance of Jenny's book [16] for the emergence and development of predictive soil mapping. At the same time, the early works of the Russian school of soil science were totally ignored.

The subject of this paper is to show that contemporary predictive soil mapping is based on the works of Vasily Dokuchaev and Sergey Zakharov.

\section{THE DOKUCHAEV HYPOTHESIS}

In 1886, Dokuchaev [9, pp. 352-353] formulated the following hypothesis (Fig. 1):

\{Any ... soil is always and everywhere a mere function ${ }^{1}$ of the following factors of soil formation: (1) the nature (content and structure) of the parent rock; (2) the climate of the given terrain; (3) the mass and character of vegetation; (4) the age of the terrain; and, finally, (5) the terrain topography. It immediately follows that (a) if the mentioned factors are the same in two different localities (however far apart they might be), the soils in the two localities should also be similar, and vice versa; consequently, (b) if we have thoroughly studied these factors, we may predict in advance what the soil itself should be like. Next, (c) it is well known that the momentum should not change if one force component increases or decreases by some value, while another force component changes by the oppo-

\footnotetext{
${ }^{1}$ The italicized text was highlighted by Dokuchaev.
} 


\section{MATEPIAЛЬЫ \\ КЪ ОЦЂНКち ЗЕМЕЛЬ НИЖЕГОРОДСКОЙ ГУБЕРНІИ.}

ЕСТЕСТВЕННО-ИСТОРИЧЕСКАЯ ЧАСТЬ

\section{ОТЧЕТЪ}

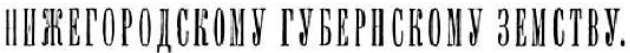

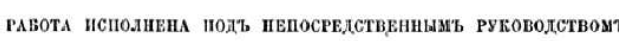

Проф. СПб. университета В. В. ДОКУЧАЕВА.

Выпусқъ I.

ГЛАВНЫЕ МОМЕНТЫ

В' НСТОРІИ ОЦБНОКЪ ЗЕМЕ.ІБ ЕВРОIЕИСКОГ РОССИ.

СЪ КЛАССИФИКАЦІЕЙ

Р У С C ह И Х Ђ ПП О प В Ђ.

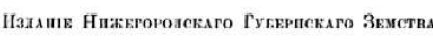

C.-ПЕТЕРБУРГЪ

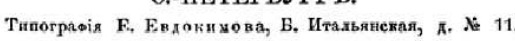

1886.
352

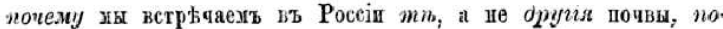

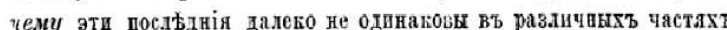

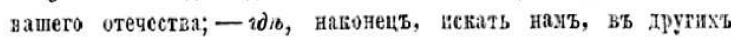
странатъ, - почвы, аналогичныя нашіми?

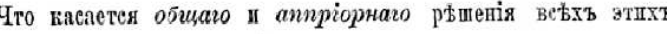

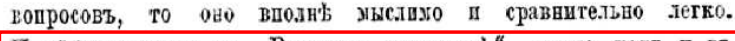

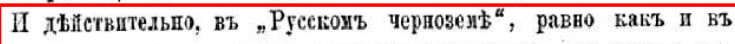
предъндущихъ главахъ, хқ старахшсь установить, уто веякая рaстительно-наземная почва всегда п всюду явлтетел простої фбункиіей отъ с.льдующшх' почвообразователей: 1) характера (составъ и етроеніе) материнской горной породв, 2) к.пшмата данной мветностп, 3) массы и характера растительностп, 4) возраста

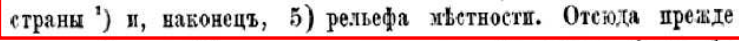

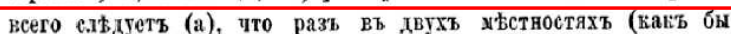

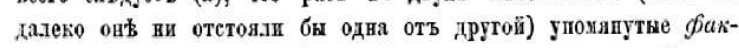

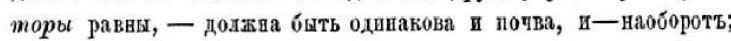

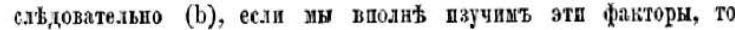
үае жалередь можсио предсказать, какова домжска бымь и jа

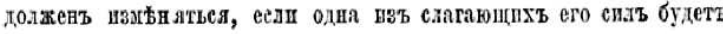

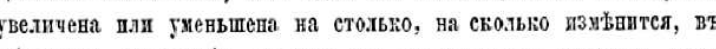
обратпояъ отнопеніп, хругая нзъ стагающихъ; понятно, тоже самое соотвошеніе доньно стңествовать, до изөнстаной степени,

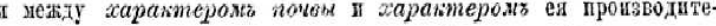
лей. Отерда ведо, что еъ теоретпческой точви зрђнія авляетея

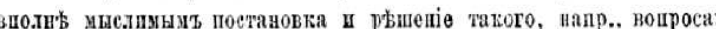

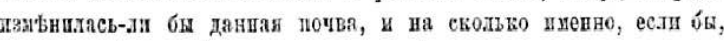

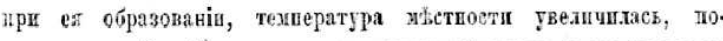

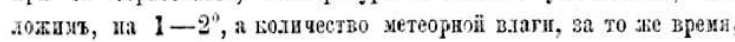

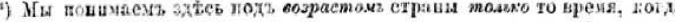

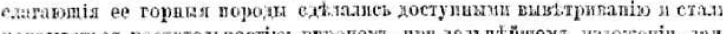

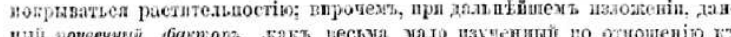

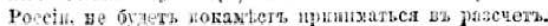

353

возросло бв на 1-2, дюйуа? Изжђнидась-лп бы почва, еелшбь пршростъ данної растительностп эвеличплея на 20 пудовъ (на теслтпну), а температгра понизшассь бн па $1-2^{\circ}$ ?

Повторяю, вее это такт ясно, такъ лотіческіп загондо и даже вецябъано, уто, въроятно, спорить противъ этого жикмо не станетъ.

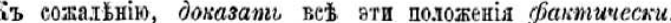

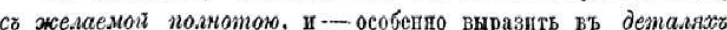

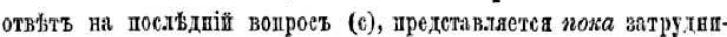

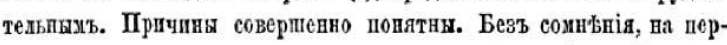

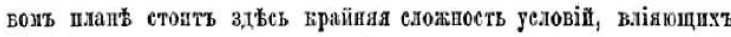
на почву; во-вторыхъ, этп уеловія не представляютъ постолпвыхъ ведичпгъ, а поэтому и трудно поддаютея дафровощу обовначенію; ваконедъ, по одниуъ пзэ үпонянутыхъ факторовъ у пасъ мало

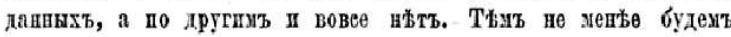
надызтьея, что І эти прешатетвіл современезъ јетранятел, пг тог,

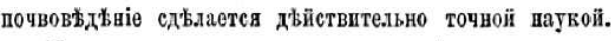

Но так'ъ Бакъ теоретическал върность вышеупомянутьхт.

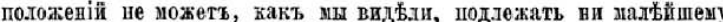
сомнЊнію, то сейчпасъ упомянутыя фактическія затрудвенія нө доджнк остапавливать насъ отъ попьтпки, по крайней хърђ,

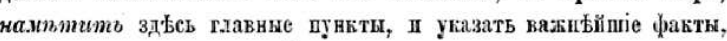
готорые, по нашему эвьпію, виоль's подтверждають висказинное

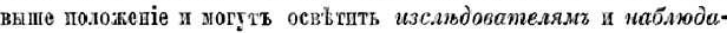

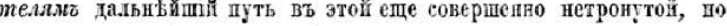
весьуа много ойъщающей обхасти...

Eлимату. Кагт зальчепо выпте, в.іяніе влпмата па обра-

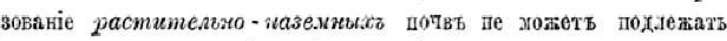

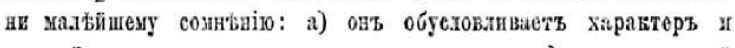

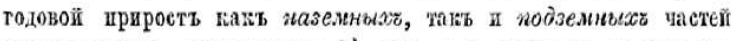

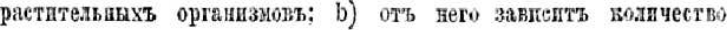

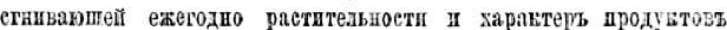

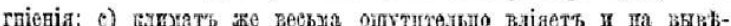
триваніе натеринеквхъ иородъ, и при тонъ не только яехангчески,

Fig. 1. Title page and pages from book [9] with the Dokuchaev hypothesis. 


\section{ДОКЛАДЪ}

\section{IPOФECCOPA B. B. ROLYYTAEBA}

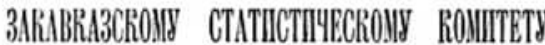

объ оцьнкь земель вообще и-Закавназья, въ особенности.

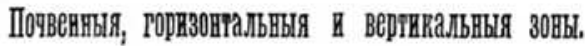

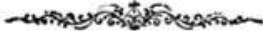

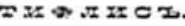

Тичограрія капџ. Гаяновау. гражд. ч. па Кавказһ, Лор.-Медик. у., д. каз. 1899.

Fig. 2. Title page and page from book [8] with the soil formation equation (3). ствз ученихъ, ато були мехажическія, тавъ сказать, случайкия смьси; о какихъ же завонахъ могла быть здъсь рьчь? Правда, какъ за границею (Ортъ, Майэръ, Лоренцъ и хруг.), такъ и у насъ (Стебутъ и друг.), били попнтки установить бодье правильвый, бодъе научньй взглядъ на почвы, но онь, кавъ это подробно разсмотрьно мвою въ другомъ мьсть ${ }^{1}$ ), почти всегда смБшивали почви съ разваго рода рыхлыми коренвнми горвыми породами и классифицировали ихъ (почвы) нли по петрографическому характеру, нли по геологическому возрасту подпочвы, забывая при этонъ, что подпочва, иначе, груктз-лишь одинъ, и то дадеко нө всегда важвьйшій, изъ почвообразователей. Мы увидимт низе, что въ Закаввазьн, вакъ и повсюду, нерђдво ва оджожз и помь же грумти, но при разныхт влиматическихъ и раститедьньхъ условіяхъ, получаютсл такія развохарактервыл почвы, вавъ краскоземы, блиоземь и чернояемь.

Несохвђнно руссвияъ почвовђдамъ принадхеаить честь уставовви осковз чистой кауки Почьовпдюнія: они первке уставовили тотвое и истиввое понятіе о почвь, какъ о внолиь самостоятельномъ, естественво-историческомъ, тьль, такомъ-же тьль, капъ и пюбое зивотное, кақъ и всявое растеніе, какъ любой минералъ. Подобно этимъ посльднимъ, и дюбая кормалькая почва, дежащая на иьсть своего происхожденія, додава ихьть свой опредьленньй гевезисъ, свое, строго опредъленное, распростравеніе, свой составъ и физику, при чемъ все әто долаво быть вавономьрно и находиться между собою въ тисктйиеей гекетической свази. Я говорю, что почва есть самостодтедьное өстественно-историческое тыло, щменно-продуктъ совокушной дыятедьности сдьдующихз фавторовъ- почвообразоватеяей: а) климата данной страны, әто важньйmiй изъ почвообразователей, ь) раститехькъхъ и животкыхә организмовъ и, нагонецъ, с) тьхъ горныхъ породъ, на готорьхъ дежитъ въ настоящее время почва, тавъ-наз. материн: скихз горнихъ породъ ${ }^{2}$ ), иначе говоря, почва-әто фуккиів отъ всђхъ вытеупомянутихъ величинъ, такъ что, если мы обозначимб почву черезъ $I$, кикмать черезъ $K$, оргакизмы $-O$, грунты $-\Gamma$,

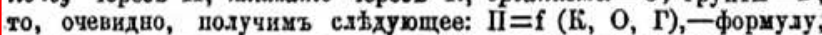
воторая ясво показываетъ усдовія образовакія, тожсдества и воз-

1) Довучаевъ. Обзоръ говньйщихъ почвеннкхъ кхассифигапій. Ма $1886 \mathrm{r}$.

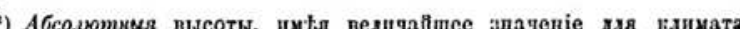

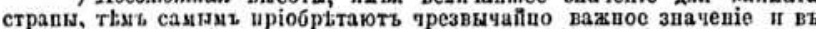

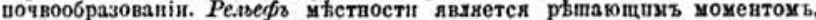

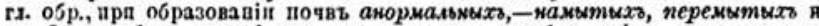
ир. Само соб́ою разумћетсл, что и ночвы, подобно всћмъ организмамъ, могуть бать цежду собою сравниваемы дипь при условіи оджого и толо

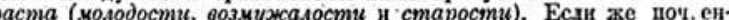

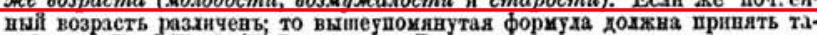
вюी вндъ $\mathrm{Il}=\mathrm{f}(\mathrm{K}, \mathrm{O}, \mathrm{\Gamma}) \mathrm{B}$, првчещъ $B$ означаеть возрасть почвы. here should be a similar, to site value. Thus, there extent, relationship between the character of the soil and the character of its forming factors. Hence, it is clear that it is theoretically possible to state and solve, for example, the following problem: How would a given soil change if there were an increase of the terrain temperature by, say, $1-2^{\circ}$, with a synchronous increase of meteoric water by $1-2$ inches? How would a soil change if there is an annual increase of the vegetative mass by 20 poods per desyatina with a decrease of the temperature by $1-2^{\circ}$ ?

Again, all these are so clear and so logically legitimate and necessary that nobody will probably argue with this.

${ }^{2}$ Pood is the unit of weight of the Russian Imperial measurement system, 1 pood $=16.38 \mathrm{~kg}$; desyatina is the unit of area of the Russian Imperial measurement system, 1 desyatina $=1.0925$ ha.
For now, unfortunately, it is difficult to prove all these propositions using facts and with desirable completeness, particularly to answer the last question (c) in detail. The reasons are quite obvious. Firstly, there is a great complexity of conditions affecting soils; secondly, these conditions have no absolute values, and, therefore, it is difficult to express them numerically; finally, we possess very few data for some factors and none whatever for others. Nevertheless, we may hope that all these difficulties will be overcome with time, and then, soil science will truly become an exact science . $^{3}$

The Dokuchaev hypothesis has the classic three-part structure of a scientific hypothesis, i.e., "postulate - suggestion - limitation." Part I of the hypothesis (the first

\footnotetext{
${ }^{3}$ This translation of the Dokuchaev hypothesis was first published in the author's book [12, pp. 167-168].
} 
УЧЕБНЫЕ РУКОВОДСТВА ДЛЯ ВЫСШЕЙ ШКОЛЫ

Проф. С. А. ЗАХАРОВ

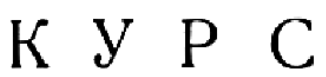

ПОЧВОВЕДЕНИЯ

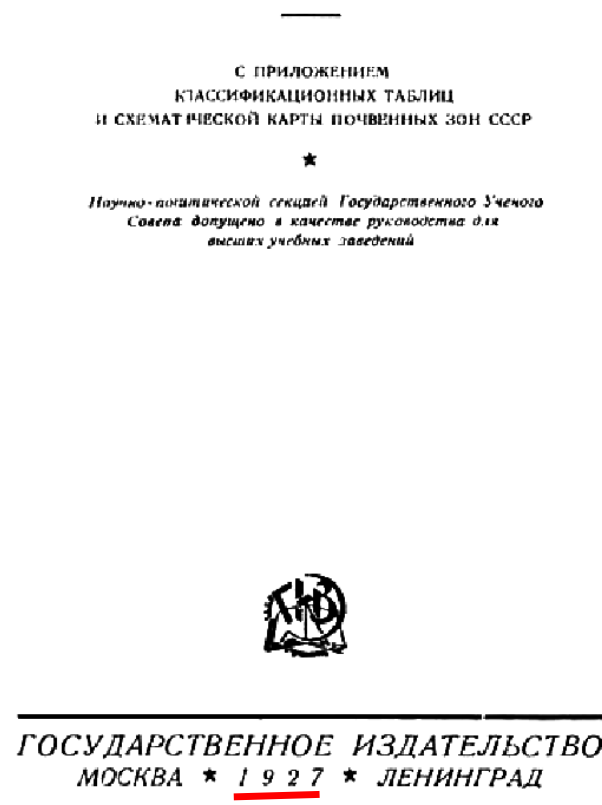

8

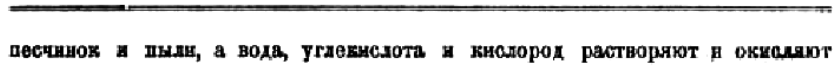

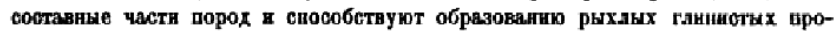
дүктов выветрнванвя. Этв-то продукты и олужат неносредственшо уатервалом сия образования почв.

Перечнслевнье природные факторы: 1) растительность в живетныс, 2) атиосферине агентв (тепло и влага) в 3) катерипские горике породы. воторне прннимают участве в образования почв, получнли название факто-

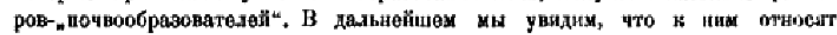
өпе также рельеф и „возраст страны“ (время).

ПІодобике же наблюдения на обширной территории русской равнишы \$али возможность проф. Дохучлесу в 80-х годах сфоржулировать, вак он

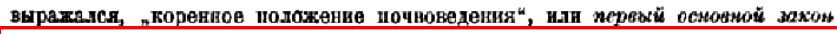
современного генетического почвоведения: „IГочва есть резуньмам совокумноё

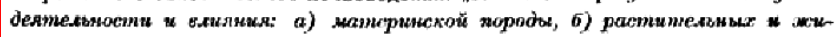

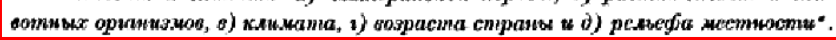
Другами словами, почвв представляет пронзводное, яли функцию, от перечисленних выше почвообразователей.

$$
\pi=t \text { (M. r. t., P. Ж. Opr. K.1.), Bo3p. เтр.. P.ф). }
$$

Из даниого закона и пз приведенной выше аксномы вытекант дочти все дальнейшие основнше положения отдельных частей почвоведения.

Почвообравованяе виражается в днфферевцировке почвенноя шасоы на генетическне горизовты. В чем же, ва основакин вашнх наблюдентй, сказывается процесс почвообразования?

Почрообразование прежде всего вкражается в накоплении перегнойных веществ в образовавни верхнего перегнойного поризонта $A$, в котором, как мы узнаем дальпе, вместе с перегноем накопляютея благодаря деятельности раетенй зольнье элөменты, - пронсходит их „аккумуляди“ (accumulatio -

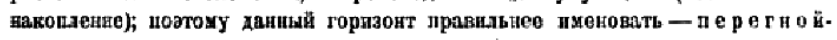
ио-акв у у дя тивния торвзонтох $A$.

Гдубяе идет горизонт, из которого совершается вымываняе иекогорых веществ, например извести иаи окисп хедеза, п в котором обыкновенио постепено ослабенает перегнойная окраска, заменяясь цветами болеө г.лубоквх слоев; зтот торнзонт получвл названве торнзовта "вымываңня" илы

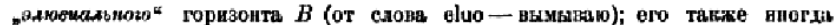
назьвант "пересодиым иоризонток $B^{\circ}$.

Еңе глубже идет сдой, в вотором мы набдюдаем сконденье некоторых вөцеств, вымытых из верхних горнзонтов $A$ п $b$, в внде скоплений угаекнслоң извести илі же соедннепий келеза. За снов характерные особенности

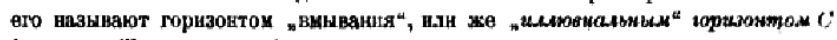
(от алова illuo - вмкваю).

Перечисленные трн торизонта находитан межлу собой в генетической

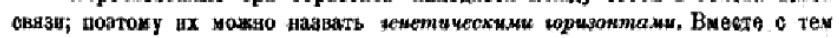

Fig. 3. Title page and page from book [35] with the soil formation equation (4).

sentence of the quotation) includes the definition of soil as a function of the five soil forming factors. This postulate, or "the basic law of soil science" [35, p. 8], was first published in complete form in 1883 , three years before the publication of the hypothesis [10]. Part II of the hypothesis (the rest of the first paragraph of the quotation) includes three main suggestions. Part III of the hypothesis (the two latter paragraphs of the quotation) includes limitations to prove the hypothesis, which were critical for the late 19th century.

From the hypothesis, one can see that Dokuchaev explicitly defined the central idea and statement of the problem of contemporary predictive soil mapping in the year 1886. It is hardly necessary to argue that the Dokuchaev hypothesis underlies, in one way or another, all modern approaches of predictive soil mapping and most of the mathematical models used in soil science. For example, Homosoil, a method for extrapolation of soil data [23] is in fact the direct implementation of points (a) and (b) of the hypothe- sis. In the last decades, the statement of the problem in point (c) of the hypothesis has been in the focus of soil scientists studying the impact of the probable climatic change on soils.

At the same time, except for Part I (a postulate familiar to any soil scientist), the Dokuchaev hypothesis was forgotten. As far as we know, Part II was last cited 84 years ago [1, p. 10], and Part III was last cited 70 years ago [16, p. 17]. Unfortunately, the Dokuchaev hypothesis was not even mentioned in national fundamental studies on the history of soil science $[30,21$, $14,6]$.

\section{SOIL FORMATION EQUATIONS}

In 1899, Dokuchaev [8, p. 3] (Fig. 2) carried out the first step towards the formalization of the problem. He proposed the first soil formation equation:

$$
\Pi=f(\mathrm{~K}, \mathrm{O}, \Gamma) \mathrm{B},
$$


where $\Pi$ is soil, $\mathrm{K}$ is climate, $\mathrm{O}$ is organisms, $\Gamma$ is parent material, and B is the age of the soil. Topography was not included into the expression probably due to a stenographer's mistake, because Eq. (3) is preceded by two sentences discussing the important role of topography in soil formation (Fig. 2).

In 1927, Zakharov presented a general soil formation equation in a well-known fundamental textbook $[35$, p. 8$]$ (Fig. 3). This equation ideally described the Dokuchaev postulate:

$$
\pi=f(\text { М.Г.П.,Р.Ж.Орг., Кл., Возр.стр.,Р-ф), }
$$

where $\pi$ is soil, М.Г.П. is parent rock material, Р.Ж.Орг. is plant and animal organisms, Кл. is climate, Возр. стр. is the age of the terrain, and P- $\phi$ is topography. There were three misprints in Eq. (4): two commas were missed and there was an excess close bracket (Fig. 3). The misprints were fixed in the second edition [36, p. 18]. Unlike the rare brochure [8], textbooks $[35,36]$ were widespread $(4000$ and 18000 copies of the first and second editions were printed, respectively).

In the summer of 1927, the Dokuchaev hypothesis and Eq. (4) became known in the West due to two circumstances. First, Afanasiev [1, p. 10] printed an English translation of almost the entire quotation cited above (without referencing book [9]) in the reprint of his report on the First International Congress of Soil Science held in Washington DC in June 1927. Second, Zakharov took part in the Congress. The reprint of his report to the Congress [34] did not include Eq. (4). We do not know the exact publication date of book [35]; therefore, we cannot state that Zakharov was able to present the book at the Congress or to give it to other participants. However, although the first review and information on this book were published in early 1928 [11], one of them was printed in the congress's proceedings [31, p. 33]. This is indirect evidence that Zakharov could have brought at least the proofs of the book to the Congress. Many western soil scientists, including Jenny [31], had an opportunity to learn about this equation in personal contacts with Zakharov during the Congress and the transcontinental soil excursion. It is well known that the very active exchange of scientific ideas between the participants from different nations was an outstanding feature of this congress [32]. The unique role of the Soviet delegation, which included several direct students of Dokuchaev, was acknowledged almost immediately [20]. One of the outcomes of this communication was a series of soil formation equations, such as the Shaw equation, the Wilde equation, and the Jenny equation (Eq. (2)).

In 1930, Shaw published the following equation [28, p. 244]:

$$
S=M(C+V)^{T}+D
$$

where $S$ is soil, $M$ is parent material, $C$ is climate, $V$ is vegetation, $T$ is time, and $D$ is erosion or deposition. Shaw did not mention either the Dokuchaev hypothesis or the equations of Dokuchaev (3) and Zakharov (4). It is interesting that Shaw presented Eq. (5) at the Second International Congress of Soil Science held in the USSR in 1930 [27]. Zakharov took part in the discussion of this presentation. He criticized Shaw for ignoring the role of topography, animals, and humans in Eq. (5) [27, p. 14].

In 1941, Wilde published his equation [33, p. 34]:

$$
S=\int(\text { g.e.b. }) d t,
$$

where $S$ is soil, $g$ is geological substratum, $e$ is the environmental influences, $b$ is the biological activity, and $t$ is time. Wilde did not mention Eqs. (3), (4), and (5). Note that Wilde, a Russia-born immigrant, was familiar with the works of Dokuchaev and Zakharov. Indeed, he cited the Dokuchaev postulate right before Eq. (6) calling it "the first basic law of soil science" (Zakharov's expression [35, p. 8]).

In 1941, Jenny published Eq. (2) [16, p. 16] and selectively cited the Dokuchaev hypothesis (after Afanasiev [1, p. 10]), that is, only a portion of the limiting Part III of the hypothesis [16, p. 17]. The following fact is more indicative: it is obvious that the Jenny equation (2) is almost identical to the Zakharov equation (4). However, Jenny did not mention Zakharov either in [16] or in subsequent papers [15, 17]. Jenny began to cite the Dokuchaev equation (3) only in 1961 [15], after its reproduction in the Collection of Works of Dokuchaev [7].

This year marks the 70th anniversary of the publication of Jenny's book [16]. During these years, the question of authorship of the soil formation equation was tangled due to several circumstances. First, the Zakharov equation (4) was forgotten. As far as we know, it was never cited both in foreign [29] and national studies of the history of soil science $[6,14,21$, 30]. Second, the Dokuchaev equation (3) was repeatedly quoted with errors $[14$, p. $61 ; 26$, p. 296]. Third, different authors proposed different precursors of the Jenny equation. For example, Bockheim et al. [4, p. 26] stated that the Shaw equation (5) was the first soil formation equation.

Finally, there was the constant problem of the incorrect citation of primary sources. For instance, Jenny repeatedly wrote that the Dokuchaev equation "was printed in 1898 in an obscure Russian journal" $[15 ; 17$, p. 203], though, in fact, Eq. (3) was printed in 1899 in brochure [8]. In the second edition of Pedology, Joffe noted the following [19, p. 124]: "In mathematical form, the Dokuchaev proposition was expressed as follows:

$$
S=f(p m, c, b, a, \text { and } t),
$$

where $S=$ soil, $f=$ function, $p m=$ parent material, $c=$ climate, $b=$ biosphere, $a=$ age of land (time factor), 
Chronology of events related to the Dokuchaev hypothesis

\begin{tabular}{c|l}
\hline Year & \multicolumn{1}{|c}{ Event } \\
\hline 1883 & The Dokuchaev postulate \\
1886 & The Dokuchaev hypothesis \\
1899 & The Dokuchaev equation \\
1927 & The Zakharov equation \\
& Translation of the Dokuchaev hypothesis into English \\
1930 & The First International Congress of Soil Science \\
1941 & The Shaw equation \\
& The Wilde equation \\
2003 & The Jenny equation \\
SCORPAN model
\end{tabular}

and $t=$ topography." Joffe did not cite a source for Eq. (7) that has a similar structure with the equations of both Zakharov (4) and Jenny (2). It is interesting that the first edition of Pedology [18], which was repeatedly cited by Jenny [16], did not include this sentence and Eq. (7). It is well known that Joffe, a Russia-born immigrant, was a supporter of the Russian school of soil science. Probably, in the second edition [19], which was published eight years after Jenny's book [16], Joffe wished to protect the scientific priority of Dokuchaev and Zakharov in the derivation of the soil formation equation. However, in terms of citation, he did this incorrectly and so increased the confusion.

As a result, many Western soil scientists ignore, as a rule, analogues or precursors of Eq. (2) and acknowledge Jenny as the author of this equation. The apology of the imaginary scientific priority of Jenny can be found elsewhere [3, 2]. Most national soil scientists believe that Jenny adopted Eq. (2) from Dokuchaev. This is also wrong because the structure of Eq. (2) differs significantly from the structure of Eq. (3). Sometimes, the expression "the Dokuchaev-Jenny equation" is used [25], but this is also incorrect because the structures of Eqs. (3) and (2) are different. For the same reason, it is incorrect to call Eq. (2) "the Dokuchaev-Zakharov equation."

Equation (2) should be called the Zakharov equation. It is encouraging that this issue was clarified in 2011, the anniversary year for the publications of Dokuchaev and Jenny (table).

\section{CONCLUSIONS}

(1) The central idea and statement of the problem of the contemporary predictive soil mapping were explicitly defined in the Dokuchaev hypothesis in the year 1886.

(2) The equations of Dokuchaev (3) and Zakharov (4) became the first experience in mathematical formalization in soil science and laid the foundation for mathematical modeling in this science. Dokuchaev and Zakharov themselves (like most of their contemporaries) did not pay much attention to these equations. Being written too early, they were finally appreciated in the last third of the 20 th century.

(3) Jenny adopted the soil formation equation from Zakharov. Equation (2) should be called the Zakharov equation.

\section{ACKNOWLEDGMENTS}

I would like to thank J.L. Meshalkina and A.V. Ivanov (Faculty of Soil Science, Lomonosov Moscow State University, Russia); B. Minasny (Faculty of Agriculture, Food, and Natural Resources, University of Sydney, Australia); I.V. Ivanov (Institute of Physical, Chemical, and Biological Problems in Soil Science, Russian Academy of Sciences, Pushchino, Russia); and the anonymous reviewer for librarian assistance, discussion, and constructive criticism.

\section{REFERENCES}

1. J. N. Afanasiev, The Classification Problem in Russian Soil Science (Academy of Sciences of the USSR, Leningrad, 1927).

2. R. Amundson, "The State Factor Theory of Soil Formation," in G. Certini, R. Scalenghe, and F. C. Ugolini (Eds.), Soils: Basic Concepts and Future Challenges (Cambridge Univ. Press, Cambridge, 2006), pp. 103112.

3. R. G. Amundson, J. W. Harden, and M. J. Singer (Eds.), Factors of Soil Formation: A Fiftieth Anniversary Retrospective, SSSA Special Publication No. 33, 1994.

4. J. G. Bockheim, A. N. Gennadiyev, R. D. Hammer, and J. P. Tandarich, "Historical Development of Key Concepts in Pedology," Geoderma 124 (1-2), 23-36 (2005).

5. J. L. Boettinger, D. W. Howell, A. C. Moore, A. E. Hartemink, and S. Kienast-Brown (Eds.), Digital Soil Mapping - Bridging Research, Environmental Application, and Operation (Springer, Dordrecht, 2010).

6. G. V. Dobrovolskii, Lectures on the History and Methodology of Soil Science (Izd. Mosk. Gos. Univ., Moscow, 2010) [in Russian].

7. V. V. Dokuchaev, "Report on Land Evaluation in General and for the Transcaucasus, in Particular. Horizontal and Vertical Soil Zones," in V. V. Dokuchaev Works, Vol. 6 (Izd. Akad. Nauk SSSR, Moscow, 1951), pp. 379-397 [in Russian].

8. V. V. Dokuchaev, Report to the Transcaucasian Statistical Committee on Land Evaluation in General and Especially for the Transcaucasia. Horizontal and Vertical Soil Zones (Tipogr. kantselyarii Glavnonachal'stvuyushchego grazhdanskoi chast'yu na Kavkaze, Tiflis, 1899) [in Russian].

9. V. V. Dokuchaev, Materials on Land Evaluation of the Nizhni Novgorod Governorate. Natural and Historical Part: Report to the Nizhni Novgorod Governorate Zemstvo. Vol. 1: Key Points in the History of Land Evaluation 
in the European Russia, with Classification of Russian Soils (Tipogr. Evdokimova, St. Petersburg, 1886) [in Russian].

10. V. V. Dokuchaev, Russian Chernozem. Report to the Imperial Free Economic Society (Tipogr. Declerona i Evdokimova, St. Petersburg, 1883) [in Russian].

11. M. Filatov, "A Course of Soil Science by S. A. Zakharov (GIZ, Moscow, 1927, 440 p.)," Pochvovedenie, Nos. 1-2, 190-192 (1928).

12. I. V. Florinsky, Digital Terrain Modeling in Soil Science and Geology (Elsevier / Academic Press, Amsterdam, 2011).

13. A. E. Hartemink, A. McBratney, and M. L. MendonçaSantos (Eds.), Digital Soil Mapping with Limited Data (Springer, Dordrecht, 2008).

14. I. V. Ivanov, History of Russian Soil Science. The Development of Ideas, Differentiation, and Institutionalization. Book 1: 1870-1947 (Nauka, Moscow, 2003) [in Russian].

15. H. Jenny, "Derivation of State Factor Equations of Soils and Ecosystems," Soil Sci. Soc. Am. Proc., 25 (5), 385-388 (1961).

16. H. Jenny, Factors of Soil Formation. A System of Quantitative Pedology (McGraw Hill, New York, 1941).

17. H. Jenny, The Soil Resource: Origin and Behavior (Springer, New York, 1980).

18. J. S. Joffe, Pedology (Rutgers Univ. Press, New Brunswick, 1936).

19. J. S. Joffe, Pedology, 2nd rev. enl. ed. (Pedology Publications, New Brunswick, 1949).

20. J. S. Joffe and I. Antipov-Karataev, "American Soils as Seen by Russian Investigators," Soil Sci. 27 (2), 159166 (1929).

21. I. A. Krupennikov, History of Soil Science from the Time of Its Inception to the Present Day (Nauka, Moscow, 1981) [in Russian].

22. P. Lagacherie, A. B. McBratney, and M. Voltz (Eds.), Digital Soil Mapping. An Introductory Perspective (Elsevier, Amsterdam, 2007).

23. B. P. Mallavan, B. Minasny, and A. B. McBratney, "Homosoil, a Methodology for Quantitative Extrapolation of Soil Information Across the Globe," in J. L. Boettinger, D. W. Howell, A. C. Moore, A. E. Hartemink, and S. Kienast-Brown (Eds.), Digital Soil Mapping-Bridging Research, Environmental Application, and Operation (Springer, Dordrecht, 2010). pp. 137-150.

24. A. B. McBratney, M. L. Mendonça Santos, and B. Minasny, "On Digital Soil Mapping," Geoderma 117 (1-2), 3-52 (2003).

EURASIAN SOIL SCIENCE Vol. $45 \quad$ No. 42012
25. A. V. Nakaryakov and V. A. Shcherbinin, "On the Ecological Meaning of the Dokuchaev-Jenny Equation in Soil Reclamation Theory," in V. N. Bol'shakov (Ed.) Fundamental and Applied Ecology. Problems of Urbanization (Proc. Int. Sci. Conf., Ekaterinburg, Feb. 3-4, 2005) (Izd. Ural'sk. Gos. Univ., Ekaterinburg, 2005), pp. 239-241 [in Russian].

26. R. J. Schaetzl and S. Anderson, Soils: Genesis and Geomorphology (Cambridge Univ. Press, Cambridge, 2005).

27. C. F. Shaw, "A Soil Formation Formula," in L. J. Prassolov and D. G. Vilensky (Eds.) Proc. and Papers of the 2nd Int. Congr. of Soil Science, Leningrad-Moscow, USSR, 20-31 July 1930. Commission V-Classification, Geography and Cartography of Soils (Selkolkhozgiz, Moscow, 1932), pp. 7-14.

28. C. F. Shaw, "Potent Factors in Soil Formation," Ecology 11 (2), 239-245 (1930).

29. J. P. Tandarich and S. W. Sprecher, "The Intellectual Background for the Factors of Soil Formation," in R. G. Amundson, J. W. Harden, and M. J. Singer (Eds.), Factors of Soil Formation: A Fiftieth Anniversary Retrospective SSSA Special Publication, No. 33, 1-13 (1994).

30. D. G. Vilensky, History of Soil Science in Russia (Sovetskaya Nauka, Moscow, 1958) [in Russian].

31. S. A. Waksman and R. B. Deemer, "Participants in the Official Tour of the 1st International Congress of Soil Science through the United States and Canada," in Proc. and Papers of the 1st International Congress of Soil Science, 13-22 June 1927, Washington, USA. Transcontinental Excursion and Impressions of the Congress and of America (Am. Organizing Comm. of the 1st Int. Congr. Soil Sci., Washington, DC, 1928), pp. 22-39.

32. S. A. Waksman and R. B. Deemer, "Preface to the 5th Souvenir Volume," in Proceedings and Papers of the 1st International Congress of Soil Science, 13-22 June 1927, Washington, USA. Transcontinental Excursion and Impressions of the Congress and of America (Am. Org. Comm. of the 1st Int. Congr. Soil Sci., Washington, DC, 1928), pp. viii-ix.

33. S. A. Wilde, Forest Soils: Origin, Properties, Relation to Vegetation, and Silvicultural Management (Soils Dept., College of Agric., Univ. of Wisconsin, Madison, WI, 1941).

34. S. A. Zakharov, Achievements of Russian Science in Morphology of Soils (Academy of Sciences of the USSR, Leningrad, 1927).

35. S. A. Zakharov, A Course of Soil Science (Gosizdat, Moscow, 1927) [in Russian].

36. S. A. Zakharov, A Course of Soil Science, 2nd rev. ext. ed. (Sel'kolkhozgiz, Moscow 1931). 